\title{
Extranodal natural killer/T-cell lymphoma of the breast: a retrospective clinicopathological analysis of a consecutive 11-year case series
}

\author{
Wei Liu ${ }^{1,2+}$, Zihang Chen ${ }^{1+}$, Fanglan $\mathrm{Li}^{3}$, Wenyan Zhang ${ }^{1}$, Weiping Liu ${ }^{1}$ and Sha Zhao ${ }^{*^{*}}$ (1)
}

\begin{abstract}
Background: Extranodal NK/T-cell lymphoma of the breast (ENKTL-Breast) is rarely detected in clinical practice, and its clinicopathological features remain unclear.

Results: A consecutive 11-year (2010-2020) ENKTL-Breast case series was retrospectively analyzed. Eight cases of ENKTL-Breast were selected, with three primary and five secondary lesions, accounting for $3.5 \%$ (8/228) of all breast lymphomas. All patients were female with a median age of 46 years. Lesions presented as solid breast masses (8/8, $100 \%$ ) and were usually located in the upper outer quadrant of the breast $(7 / 8,87.5 \%)$. B-symptoms were observed in seven $(7 / 8,87.5 \%)$ cases. Two primary ENKTL-Breast cases showed concomitant diseases (IgA nephropathy and chronic active hepatitis B). Histological and immunohistochemical features of ENKTL-Breast were similar to those of ENKTL at other sites. T-cell receptor rearrangement revealed clonality in all examined primary cases $(2 / 2,100 \%)$, but only in one secondary case $(1 / 5,20 \%)$. The disease progressed rapidly in two primary cases and both patients died within 3 and 9 months. For secondary cases, the disease manifested as a disseminated disease, with a median survival time of 6 months.
\end{abstract}

Conclusions: Our data suggested that ENKTL-Breast clinically mimics breast cancer to some extent, though B-symptoms might serve as a distinguishing factor. ENKTL-Breast is highly aggressive and patients with this disease exhibit a short survival time. Primary ENKTL-Breast tends to originate from activated cytotoxic T-cells, and immune-related diseases may be involved in its pathogenesis and development.

Keywords: Extranodal natural killer/T-cell lymphoma, Breast lymphoma, T-cell lymphoma, Epstein-Barr virus

\section{Background}

Extranodal natural killer/T-cell lymphoma (ENKTL) is an uncommon malignancy, which is prevalent in East Asia and Central and South America and is highly associated with Epstein-Barr virus (EBV) [1-5]. In ENKTL, the upper aerodigestive tract is most involved, with the nasal cavity being the prototypical site of involvement. The

\footnotetext{
*Correspondence: hxblzhaosha@126.com

'Wei Liu and Zihang Chen contributed equally

1 Department of Pathology, West China Hospital, Sichuan University, No.

37, GuoXue Xiang, Chengdu 610041, Sichuan, China

Full list of author information is available at the end of the article
}

disease may also involve only extranasal sites, such as the skin, subcutaneous tissues, gastrointestinal tract, and testes. However, it rarely involves the breast [1]. In fact, only a few cases of ENKTL of the breast (ENKTL-Breast) have been reported so far [6-12]. Even in East Asia, where a higher incidence of ENKTL is observed, there are only sporadic cases of ENKTL-Breast. As a result, systematic analysis and data of ENKTL-Breast are lacking [5].

ENKTL involves different extranasal sites and presents distinct clinicopathological features depending on the primary site of involvement. For instance, gastrointestinal lesions often present with perforation or bleeding, laryngeal lesions can mimic inflammation or 
well-differentiated squamous cell carcinoma [13], and neoplastic cells of testicular lesions may show aberrant CD20 expression [14]. Thus, we hypothesized that ENKTL-Breast may have distinct clinicopathologic features that differentiate it from other involved sites; consequently, we retrospectively analyzed the clinicopathological features of eight patients with ENKTLBreast in a single-center study. We believe that this study will aid clinicians and pathologists to better recognize this rare ENKTL presentation.

\section{Results \\ Clinical features}

Eight cases of ENKTL-Breast, including three primary and five secondary cases, were selected for this study. The clinical features of these cases are summarized in Table 1. In the secondary ENKTL-Breast cases, two patients (cases 5 and 6) had a history of nasal ENKTL, one patient (case 4) had primary vaginal ENKTL, one patient (case 8) showed simultaneous involvement of the breast and gastrointestinal tract, and the remaining patient (case 7) did not have sufficient data to confirm primary ENKTL-Breast.

All patients were female, with a median age of 46 years (range 26-63 years), and most presented with B-symptoms $(7 / 8,87.5 \%)$. From the radiological results, all lesions presented as solid masses (Fig. 1a) with a median length of $2.5 \mathrm{~cm}$ (range 1.0-5.7 cm) in the greatest dimension, with five cases occurring on the right side $(5 / 8,62.5 \%)$, and seven cases located in the upper outer area of the breast $(7 / 8,87.5 \%)$. Most cases $(7 / 8,87.5 \%)$ presented with a unilateral lesion while one secondary case (case 7) showed bilateral involvement. Only one case (case 3 ) had cutaneous infiltration leading to formation of an ulcer. All patients were clinically suspected of having breast cancer at the initial hospital visit. In addition, one of the patients showed enlargement and swelling of the breast (case 1; Fig. 1b). In the laboratory tests, elevated lactate dehydrogenase levels and plasma EBV-DNA loads were detected in $80 \%(4 / 5)$ and $75 \%(3 / 4)$ of the patients who undertook the test, respectively. Seven cases were available for staging, of which three were stage I (Fig. 1c) and the other four were stage IV.

Additionally, two primary cases and one secondary case showed concomitant diseases. Case 1 (primary case) had a history of IgA nephropathy (stage IV) for 8 years, case 2 (primary case) had a history of chronic active hepatitis $B$ with abnormal liver function and elevated hepatitis B virus (HBV)-DNA load $\left(2.4 \times 10^{6} \mathrm{IU} /\right.$ $\mathrm{mL}$ ), and case 8 (secondary case) had Graves' disease for 10 years.

\section{Morphological features}

Morphologically, the primary and secondary ENKTLBreast cases were similar. The histopathological features are summarized in Table 2. All eight cases showed lymphoid cell infiltration in the mammary ducts (Fig. 2a), lobules (Fig. 2b), and stroma (Fig. 2c), and formation of lymphoepithelial lesions (Fig. 2a). An angiocentric and/or angiodestructive tumor growth pattern was also detected (Fig. 2d). Patchy or cluster necrosis $(6 / 8,75 \%)$ and apoptotic bodies $(8 / 8,100 \%)$ were observed (Fig. 2e). Neoplastic cells involved subcutaneous fat lobules, partly with septa sparing and rimed fat spaces $(8 / 8,100 \%$; Fig. 2f). Most cases $(6 / 8,87.5 \%)$ displayed apparent heterogeneity of pleomorphic tumor cells (Fig. $2 \mathrm{~h}$ ) in medium-to-large or large size, with irregular or twisted nuclei, granular chromatin, and inconspicuous or small nucleoli. Further, prominent nucleoli and vesicular nuclei were also found in some large cells. Two monomorphic cases (cases 5 and 7) were composed of small-to-medium and large cells, respectively (Fig. 2g). Mitoses were commonly observed in all cases.

\section{Immunohistochemical phenotype and molecular findings} All cases were positive for CD3 (Fig. 3a) and cytotoxic granules (TIA-1/GrB; Fig. 3e), and negative for CD20, CD5 (Fig. 3b), CD4, and CD8. CD56 (Fig. 3d) was positive in most cases $(6 / 8,75 \%)$. Six cases were positive for CD30 (median: 62.5\%; range 20-80\%; Fig. 3c). Median proliferation index $\mathrm{Ki}-67$ was $80 \%$ (range $40-90 \%$ ). Seven cases were tested for T-cell receptor $(T R)-\gamma$ rearrangement, of which two primary cases and one secondary case were monoclonal. EBV-encoded small RNAs (EBER) was positive for all cases (range 40-80\%; Fig. 3f). The abovementioned data are summarized in Table 3.

\section{Follow-up and survival analysis}

Six cases were available for follow-up and survival analysis (two primary and four secondary). For the two primary cases, although the patients were at stage I at the time of diagnosis (radiologically confirmed), the disease progressed rapidly and involved multiple organs in a short span of time. These patients responded poorly to chemotherapy and died of the disease within 9 and 3 months, respectively. For the four secondary cases, many organs other than the breast were also involved and showed wide dissemination. These patients also had a poor response to chemotherapy and/or radiotherapy, with a median OS of only 6 months (range $2-11$ months).

\section{Review of the reported cases of ENKTL-breast}

We identified seven cases of ENKTL-Breast (both primary and secondary) with sufficient clinicopathological 


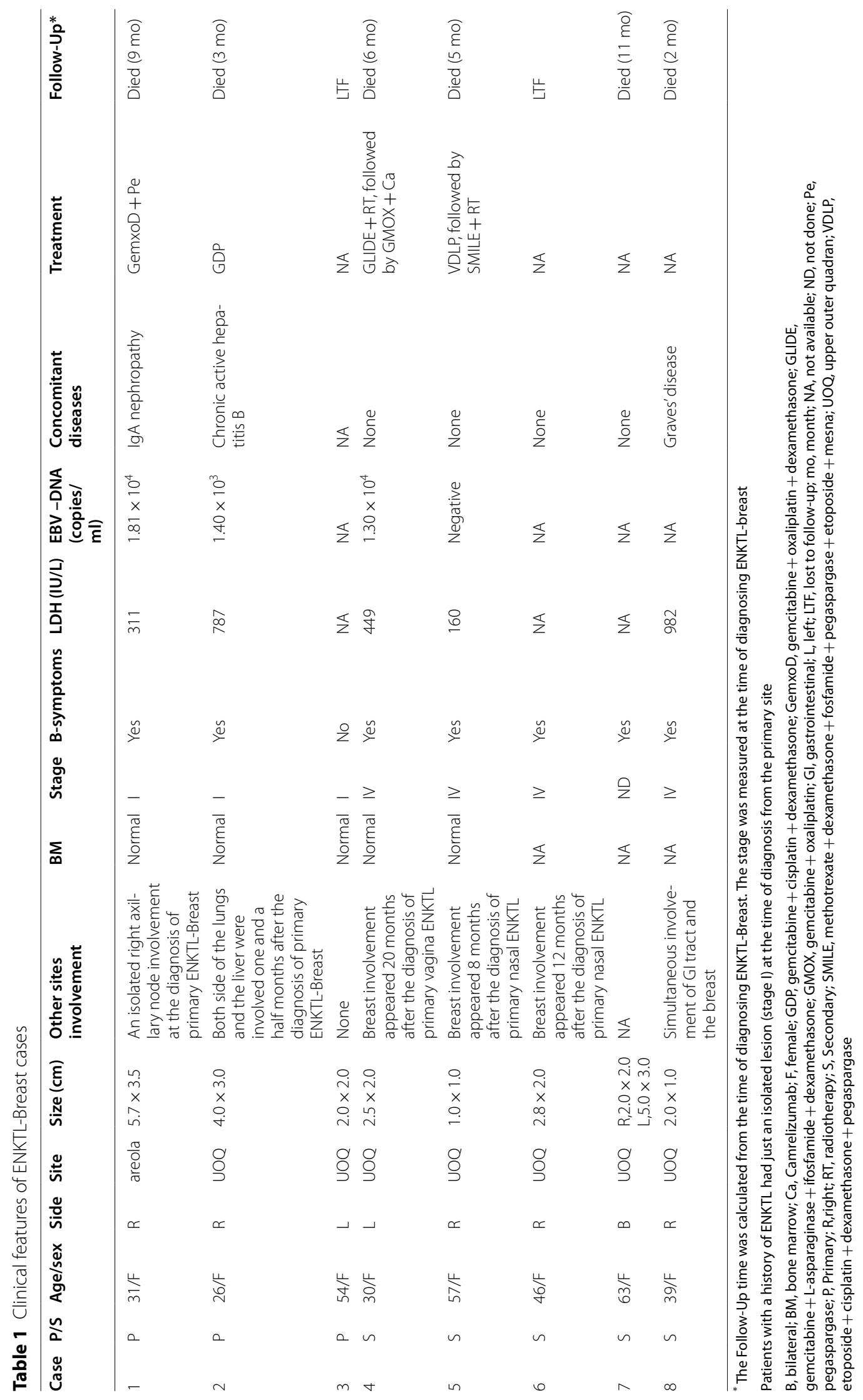




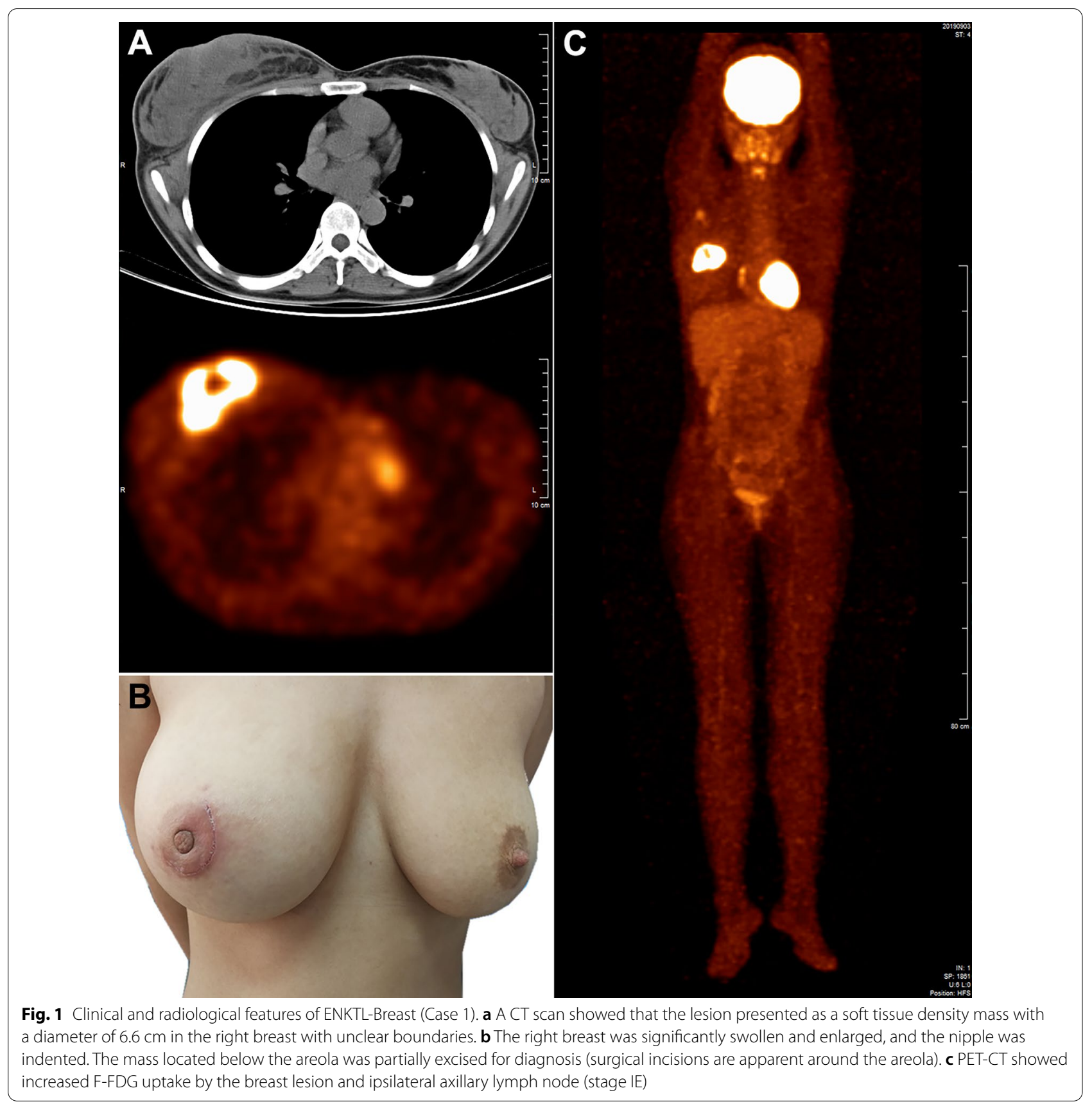

data acquired from literature review [6-11]. The clinicopathological features of these cases and those of our study are summarized in Table 4 (also see Additional file 1: Table S1, which summarizes these features in detail). A total of 15 cases (including our cohort and cases from the literature) were collected, including seven primary cases and eight secondary cases. Nine out of $12(75 \%)$ of the patients were Asian. All patients were female and had a median age of 41 years (range 20-63 years). Majority of the cases showed
B-symptoms (10/12, 83.3\%) and initially presented with a breast mass $(14 / 15,93.3 \%)$. Beside the three cases from our cohort that presented with a coexistent disease, three cases in the literature also reported heart transplantation, saline breast implants, and hypothyroidism, respectively. Compared with the secondary cases $(1 / 6,16.7 \%)$, primary cases tended to have clonal $T R$ rearrangement $(3 / 5,60 \%)$. Of the 10 patients with survival data, $90 \%$ (9/10) died of the disease, and had a median survival of 5 months. 
Table 2 Morphological features of ENKTL-Breast cases

\begin{tabular}{|c|c|c|c|c|c|c|c|c|c|}
\hline Case & Biopsy & Skin ulcer & PEH & Infiltration area & $\begin{array}{l}\text { Angiocentric/ } \\
\text { angiodestructive }\end{array}$ & $\begin{array}{l}\text { Patchy } \\
\text { necrosis }\end{array}$ & $\begin{array}{l}\text { Apoptotic } \\
\text { bodies }\end{array}$ & Cell size & Shape of cell \\
\hline 1 & Excision & - & - & Breast parenchyma & + & + & + & Medium-large & Pleomorphic \\
\hline 2 & Core needle biopsy & - & - & Breast parenchyma & + & + & + & Large & Pleomorphic \\
\hline 3 & Excision & + & - & $\begin{array}{l}\text { Breast parenchyma } \\
\text { and overlying epithe- } \\
\text { lium }\end{array}$ & + & + & + & Large & Pleomorphic \\
\hline 4 & Core needle biopsy & - & - & Breast parenchyma & + & + & + & Medium & Pleomorphic \\
\hline 5 & Core needle biopsy & - & - & Breast parenchyma & + & - & + & Small-medium & Monomorphic \\
\hline 6 & Core needle biopsy & - & - & Breast parenchyma & + & - & + & Medium & Pleomorphic \\
\hline 7 & Excision & - & - & Breast parenchyma & + & + & + & Large & Monomorphic \\
\hline 8 & Excision & - & - & Breast parenchyma & + & + & + & Medium-large & Pleomorphic \\
\hline
\end{tabular}

PEH, pseudoepitheliomatous hyperplasia; +, positive; - , negative

(See figure on next page.)

Fig. 2 Morphological features of ENKTL-Breast (hematoxylin and eosin statin). a Diffuse dense infiltration of lymphoid cells in mammary ducts and surrounding stroma, forming lymphoepithelial lesions ( $\times 100$ magnification). b Lymphoma cells infiltrated the lobules ( $\times 200$ magnification). c Tumor cells infiltrated the specialized breast stroma ( $\times 400$ magnification). $\mathbf{d}$ Angiocentric and angiodestructive growth patterns ( $\times 200$ magnification). e Patches of coagulative necrosis and apoptotic bodies are apparent ( $\times 400$ magnification). $\mathbf{f}$ Neoplastic cells involved subcutaneous fat lobules of the breast, partly with sparing of septa and rimed fat spaces ( $\times 400$ magnification). $\mathbf{g}$ Monomorphic large cells ( $\times 400$ magnification). $\mathbf{h}$ Pleomorphic tumor cells ( $\times 400$ magnification)

\section{Discussion}

Non-Hodgkin lymphoma (NHL) of the breast is uncommon, accounting for approximately $5 \%$ of all breast malignancies [15-18]. According to the literature review, the most common histological breast lymphoma type is diffuse large B-cell lymphoma. T-cell or NK/T-cell lymphomas that involve the breast are uncommon. Both primary and secondary ENKTL usually present with a breast mass and clinical findings concerning for breast cancer. Herein, we summarize clinicopathological features of ENKTL presenting in the breast. To the best of our knowledge, this is the largest cohort of ENKTLBreast reported to date.

The clinicopathological features of ENKTL-Breast cases showed many similarities between our cases and previously reported cases. We also presented the characteristics of primary ENKTL-Breast cases and ENKTL at other primary sites in Table 5. Clinically, primary ENKTL-Breast mainly occurs in young to middle-aged (median: 40 years, range 20-54 years) female patients. The age of onset of primary ENKTL-Breast was similar to those of primary extra-facial-midline ENKTL (gastrointestinal tract, skin, and testis). However, primary facial midline ENKTL (nasal and larynx) often occurred in patients older than 50 years old. The patients typically presented with a mass in the upper outer quadrant of the breast (other than ulceration); however, they were distinct from primary ENKTL at other sites, which led to the initial consideration of breast cancer. Nevertheless, primary ENKTL-Breast patients were more likely to suffer from B-symptoms, and this could be a possible clue for physicians to think of a differential diagnosis. Notably, in East Asia and Central/South America, where ENKTL is more common, physicians should be aware of this condition, since ENKTL is also one of the common types of breast lymphomas (the $4^{\text {th }}$ most common type in our institution). Interestingly, both in our cohort and in cases queried from the literature, primary cases showed immune-related concomitant diseases or conditions, including immunosuppression status (heart transplantation) [7], autoimmune diseases (IgA nephropathy, hypothyroidism) [9], and other diseases or conditions that may have affected immune function (chronic active HBV infection, saline breast implant) [8]. Similarly, several breast lymphoma case series have reported a high prevalence of antecedent autoimmune diseases, such as Hashimoto's thyroiditis $(19-30 \%)[19,20]$. In addition, an increased risk of NHL in connective tissues and autoimmune diseases has also been reported [21, 22]. It is suggested that immune-related diseases may play a potential role in primary ENKTL-Breast, although robust data of such patients are lacking. We speculate that this may be because patients with immune-related diseases or conditions are more prone to EBV infection/reactivation, and this long-lasting activation may further promote the occurrence of ENKTL. Thus, more cases and 

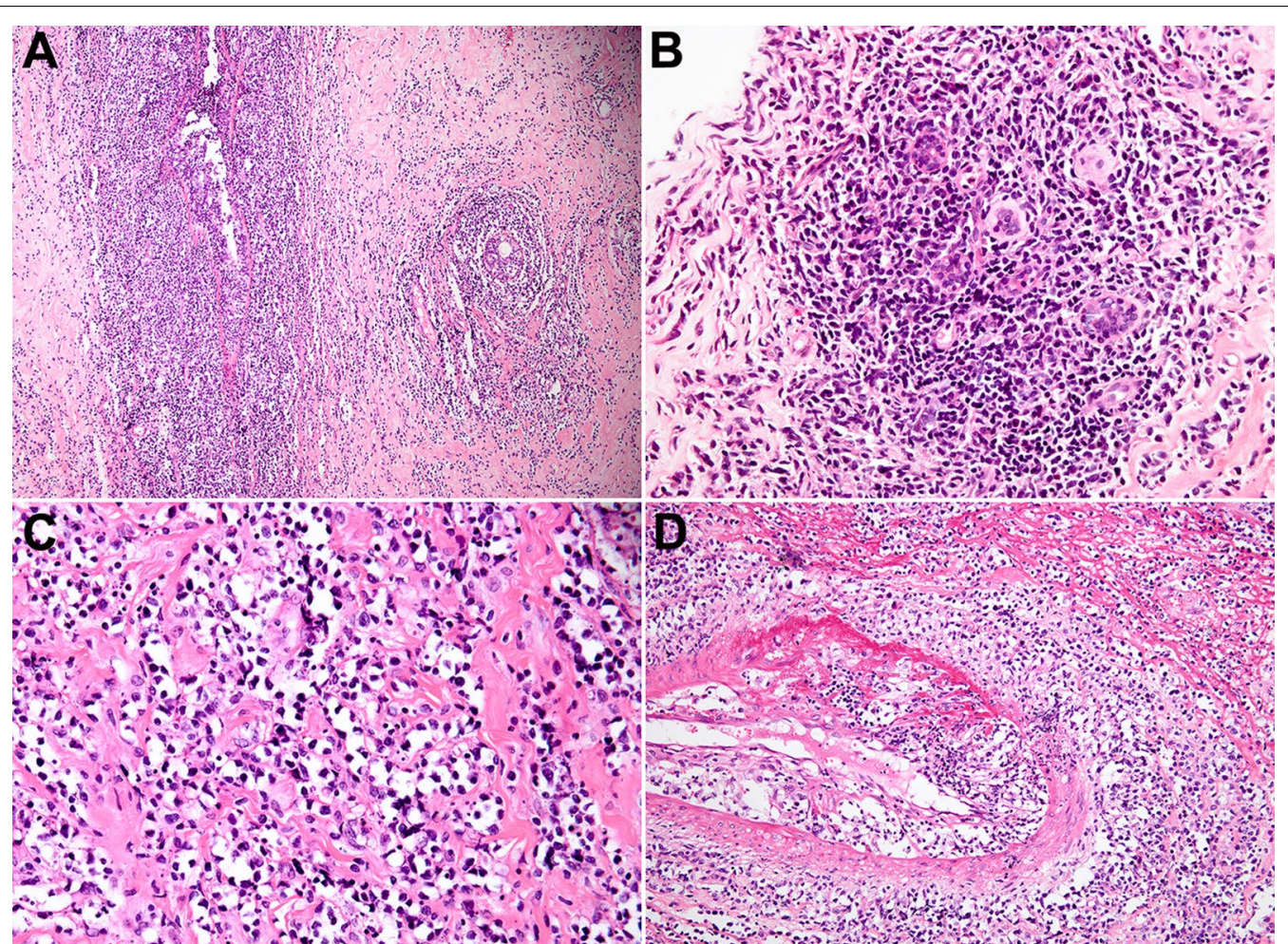

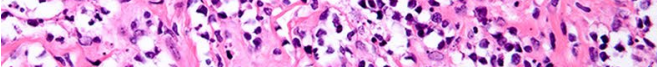
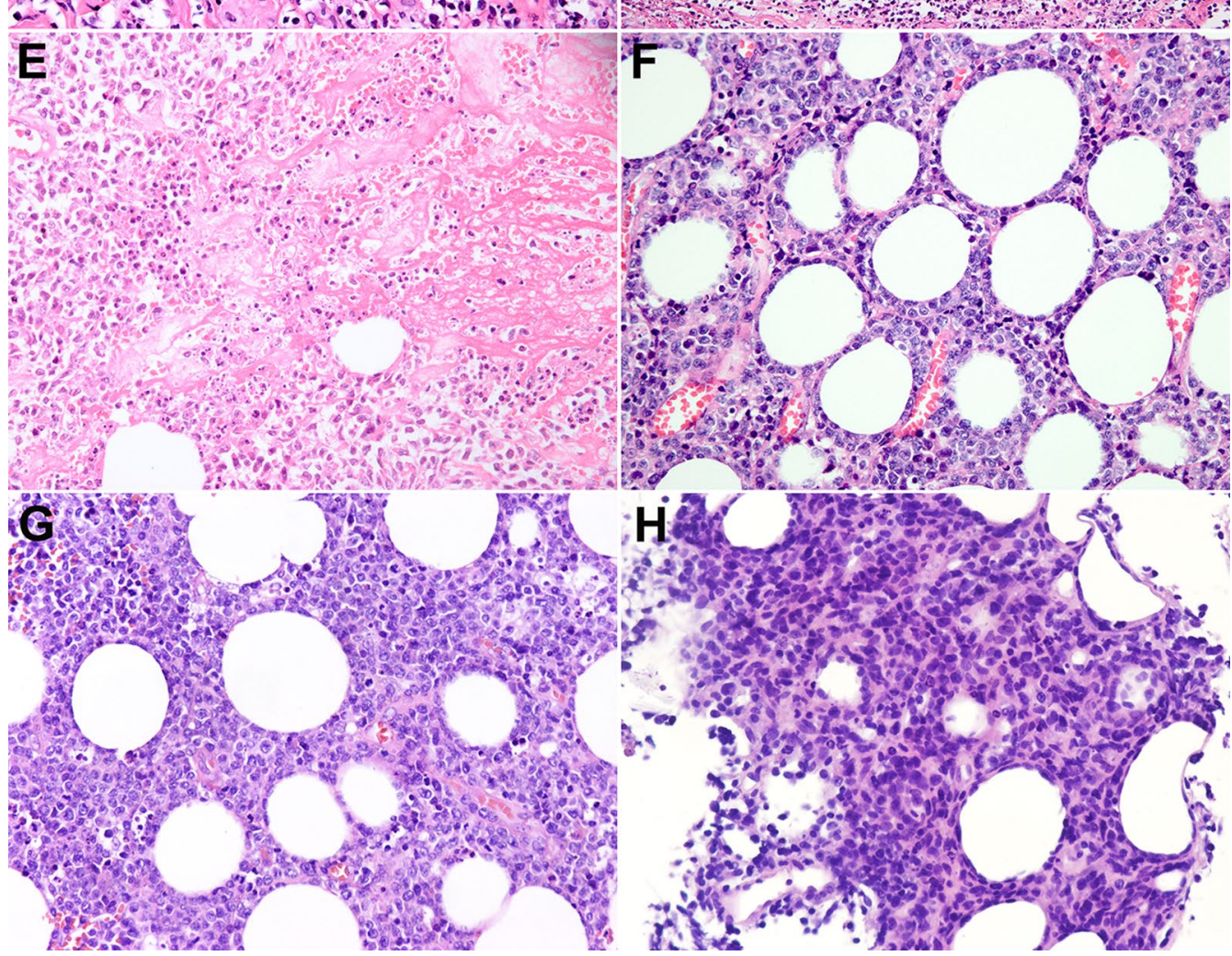

Fig. 2 (See legend on previous page.) 


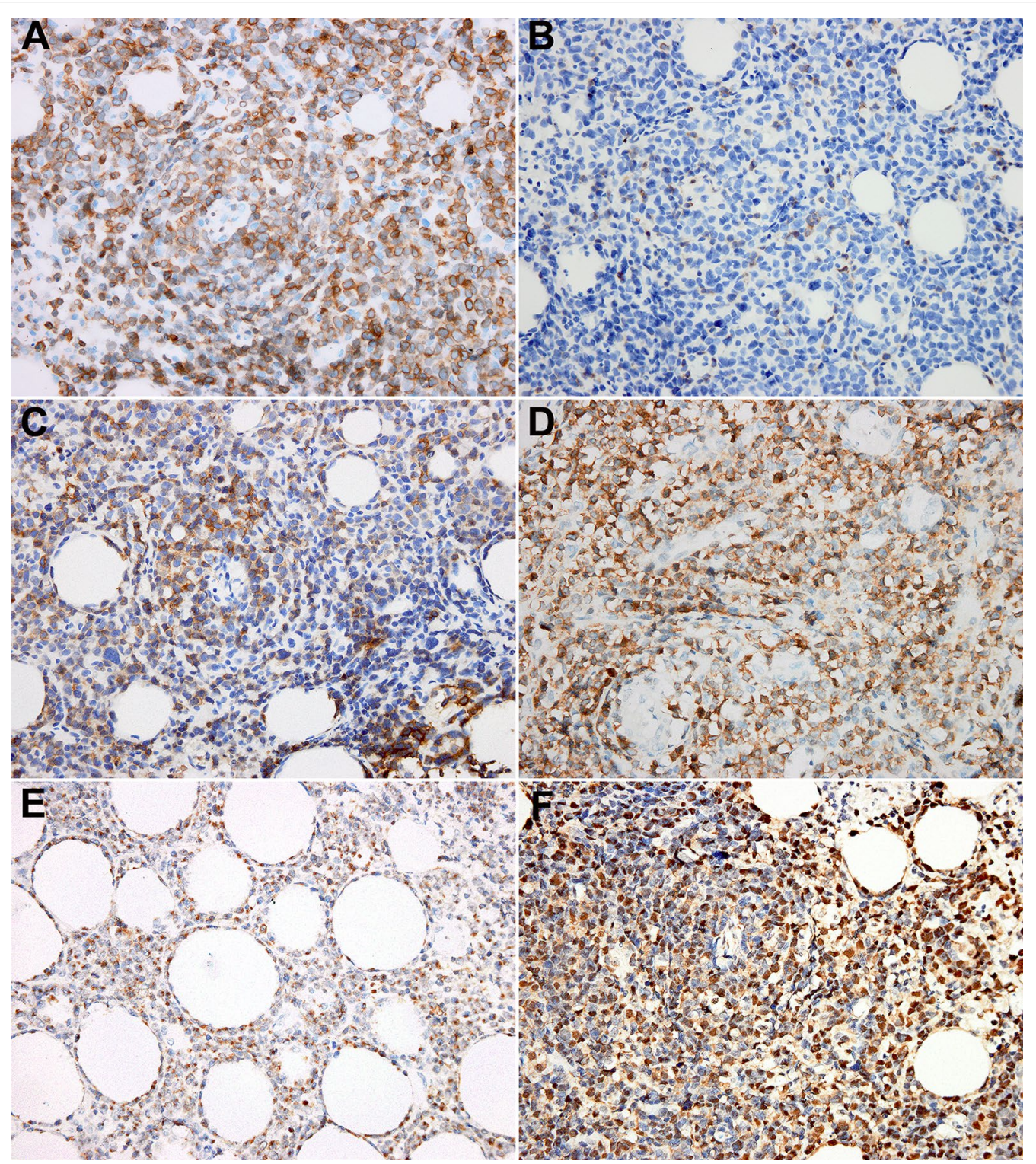

Fig. 3 Immunophenotype and in situ hybridization features of ENKTL-breast. a CD3; b CD5; c CD30; d CD56; e TIA-1; f EBER (× 400 magnification)

Table 3 Immunohistochemical analysis, EBER, and TR- $\gamma$ rearrangement study of ENKTL-Breast cases

\begin{tabular}{|c|c|c|c|c|c|c|c|c|c|c|c|c|c|c|}
\hline Case & cCD3 & CD20 & CD2 & CD5 & CD7 & CD4 & CD8 & TIA-1 & GrB & CD56 & CD30 & Ki-67 & EBER & TR- $\gamma$ \\
\hline 1 & + & - & ND & - & ND & - & - & + & + & - & $55 \%$ & $75 \%$ & + & + \\
\hline 2 & + & - & + & - & - & - & - & + & + &,+ partly positive & $70 \%$ & $80 \%$ & + & + \\
\hline 3 & + & - & ND & - & ND & ND & ND & + & + & + & $80 \%$ & $85 \%$ & + & ND \\
\hline 4 & + & - & ND & - & ND & - & - & + & + & - & $20 \%$ & $85 \%$ & + & - \\
\hline 5 & + & - & ND & - & ND & - & - & + & + & + & 0 & $80 \%$ & + & + \\
\hline 6 & + & - & ND & - & ND & ND & ND & + & + & + & 0 & $40 \%$ & + & - \\
\hline 7 & + & - & + & - & + & - & - & + & + & + & $75 \%$ & $80 \%$ & + & - \\
\hline 8 & + & - & + & - & + & - & - & + & + & + & $20 \%$ & $90 \%$ & + & - \\
\hline
\end{tabular}

cCD3, cytoplasmic CD3; GrB, granzyme B; ND, not done; TR- $\gamma$, T-cell receptor- $\gamma$ gene rearrangement; EBER, EBV-encoded small RNAs; $+:$ positive/clonal TR- $\gamma$ rearrangement; - : negative/not clonal TR- $\gamma$ rearrangement 
Table 4 Clinical features of ENKTL-Breast cases acquired from the present study and literature review

\begin{tabular}{|c|c|c|c|}
\hline Characteristics & Present study & Literature [6-11] & Total \\
\hline Number of cases & $8(P: 3 ; S: 5)$ & $7(P: 4 ; S: 3)$ & $15(P: 7 ; S: 8)$ \\
\hline Median age (range) (year) & $46(26-63)$ & $38(20-47)$ & $41(20-63)$ \\
\hline Origin (Asian/non-Asian) & $8 / 0$ & $1 / 3$ & $9 / 3$ \\
\hline Initial presented with breast mass (yes/no) & $8 / 0$ & $6 / 1$ & $14 / 1$ \\
\hline B symptoms (yes/no) & $7 / 1$ & $3 / 1$ & $10 / 2$ \\
\hline Ann Arbor stage (I/II vs III/IV) & $P: 3 / 0 ; S: 0 / 4$ & $P: 2 / 0 ; S: 0 / 3$ & $P: 5 / 0 ; S: 0 / 7$ \\
\hline Immunophenotype CD56 (positive/negative) & $P: 2 / 1 ; S: 4 / 1$ & $P: 2 / 0 ; S: 2 / 1$ & $P: 4 / 1 ; S: 6 / 2$ \\
\hline EBER (positive/negative) & $8 / 0$ & $6 / 1$ & $14 / 1$ \\
\hline TR gene rearrangement (monoclonal/polyclonal) & $P: 2 / 0 ; S: 1 / 4$ & $P: 1 / 2 ; S: 0 / 1$ & $P: 3 / 2 ; S: 1 / 5$ \\
\hline Concomitant diseases (yes/no) & $P: 2 / 0 ; S: 1 / 4$ & $P: 3 / 0 ; 5: 0 / 3$ & P: $5 / 0 ; S: 1 / 7$ \\
\hline Outcome(died/alive) & $6 / 0$ & $3 / 1$ & $9 / 1$ \\
\hline Median survival (range) (month) & $6(2-11)$ & $5(1-18)$ & $5(1-18)$ \\
\hline
\end{tabular}

NA, not available; P, primary cases; S, secondary cases, mo, months; EBER, EBV-encoded small RNAs; TR, T-cell receptor

Table 5 Clinicopathological features of ENKTL of different primary sites

\begin{tabular}{|c|c|c|c|c|c|c|}
\hline Characteristics & Breast*, n (\%) & Nasal, n (\%) [23] & Gl tract, n (\%) [24] & Skin, n (\%) [25] & Testis, n (\%) [14] & Larynx, n (\%) [13] \\
\hline Number of cases & 7 & 92 & 55 & 16 & 21 & 31 \\
\hline Median age (range) (year) & $40(20-54)$ & $52(21-89)$ & $39(14-75)$ & $32(16-72)$ & $44(21-79)$ & $50(13-77)$ \\
\hline B symptoms & $5(100)$ & $36(39)$ & $19(35)$ & $11(69)$ & $7(33)$ & $10(32)$ \\
\hline Ulceration & $0(0)$ & $N R$ & $55(100)$ & $7(44)$ & NR & $12(39)$ \\
\hline Concomitant diseases & $5(100)$ & NR & NR & NR & NR & NR \\
\hline Pleomorphic & $5(83)$ & NR & $42(76)$ & $13(81)$ & NR & $8(26)$ \\
\hline Number of CD30 + cases & $4(67)$ & $36(39)$ & $13(41)$ & $2(15)$ & $5(31)$ & NR \\
\hline Median CD30 expression, \% (range) & $62.5(50-80)$ & NR & NR & NR & NR & NR \\
\hline Median Ki-67 index, \% (range) & $80(70-95)$ & NR & $70(50-90)$ & $60(N R)$ & $80(50-90)$ & $60(30-80)$ \\
\hline $\begin{array}{l}\text { TR gene rearrangement (monoclo- } \\
\text { nal/polyclonal) }\end{array}$ & $3(60)$ & $35(38)$ & $9(53)$ & $0(0)$ & NR & $1(17)$ \\
\hline Median survival (range) (month) & $5(1-9)$ & $19.2(\mathrm{NR})$ & $14(1-56)$ & $7(2-20)$ & $15.3(0.5-87)$ & $9(1-104)$ \\
\hline
\end{tabular}

* Including primary cases from the current study and the literature review in Additional file 1: Table S1

NR, not report; TR, T-cell receptor; $n$, number

fundamental research are needed to clarify the relationship between immune-related diseases or conditions and primary ENKTL-Breast.

Morphologically, pleomorphic tumor cells diffusely infiltrate the breast parenchyma, similar to nasal and extranasal ENKTL. In all our cases, the neoplastic cells were positive for CD3 and TIA1, with a high Ki-67 index (median: 80\%, range 70-95\%) and negative for CD5, in accordance with other extranasal ENKTL. Primary ENKTL-Breast tended to express CD30 (4/6, $67 \%$ ) at a relatively high positive rate (median: $62.5 \%$, range $50-80 \%)$. Notably, primary lesions were likely to have monoclonal $T R$ rearrangements $(3 / 5,60 \%)$. In our cohort, neoplastic cells of two primary ENKTL-Breast cases $(2 / 2,100 \%)$ that underwent $T R$ rearrangement showed monoclonality with a cytotoxic T-cell phenotype. In addition, 33\% of the primary ENKTL-Breast cases from the literature review also reported a monoclonal $T R$ gene rearrangement [8]. Clonal $T R$ gene rearrangement has been reported in 10-40\% of ENKTL, presumably because of cytotoxic T lymphocyte origin [1]. Additionally, a large case series from the MD Anderson Cancer Center has also reported that extranasal ENKTL cases were more likely to carry monoclonal $T R$ gene rearrangements (extranasal type: $80 \%, 4 / 5$ vs. nasal type: $27 \%, 4 / 15$ ) [12]. Therefore, examining normal T-cell populations and further studying these malignancies in the breast may help in elucidating the origin and behavior of these exceedingly rare lymphomas.

In our series, primary ENKTL-Breast had a poor prognosis with a median OS of only 5 months (range 1-9 months), which is much shorter than that reported for the nasal ENKTL cohort by the International Peripheral T-cell Lymphoma Project (19.2 months) [23]. 
Moreover, its median OS was also the lowest among the extranasal ENKTL cohorts with other common sites (GI tract, skin, and testis) analyzed by both our institution and other hospitals (median OS: 7-15.3 months) [14, $24,25]$. This may have been due to the aggressive behavior of the disease, i.e., rapid dissemination to other sites and chemotherapy resistance even in patients with early stage disease. It may be argued that the disease is probably already disseminated from the beginning; therefore, a comprehensive assessment and close monitoring is required for such primary ENKLT-Breast cases. Similarly, we observed that the prognosis was poor for the secondary ENKTL-Breast cases, with a median OS of 6 months (range 2-18 months). Both our results and data from the literature review demonstrated that breast involvement implied advanced stage ENKTL with multiorgan involvement. Therefore, whole-body images for measuring disease involvement followed by effective treatment strategies are urgently needed. It is encouraging that novel therapies, such as immunotherapy (PD1/PDL1 inhibitor) and other targeted therapies (anti-CD30 therapy), have been shown to prolong the OS in relapse and refractory ENKTL cases [26-28]. Perhaps, these therapies can bring hope to patients experiencing ENKTL-Breast.

\section{Conclusions}

In conclusion, both primary and secondary ENKTLBreast cases are rare neoplastic diseases that often present with solid masses in the breast parenchyma (other than ulceration), whereby its growth pattern and morphology are similar to those of primary ENKTL at other sites. Primary ENKTL-Breast was more likely to have a monoclonal $T R$ rearrangement. Immune-related diseases and EBV reactivation may play a potential role in the pathogenesis. Moreover, both primary and secondary ENKTL-Breast cases present a highly aggressive clinical course, short survival time, and poor response to therapy. Thus, more study is needed to unravel the underlying etiology and provide new therapeutic options for patients with ENKTL-Breast.

\section{Materials and methods \\ Case selection}

In total, 228 patients were diagnosed with breast lymphoma (including primary and secondary lesions) between 2010 and 2020 based on data acquired from the database of the Department of Pathology, West China Hospital, Sichuan University. Histologically, the most common lymphoma type was diffuse large B-cell lymphoma, not otherwise specified (176/228, 77.2\%), followed by extranodal marginal zone lymphoma of mucosa-associated lymphoid tissue (MALT lymphoma; $11 / 228,4.8 \%)$, and lastly B-lymphoblastic leukemia/ lymphoma (11/228, 4.8\%) (Additional file 1: Table S2). Eight cases $(8 / 228,3.5 \%)$ of ENKTL-Breast were identified, which was the 4th most common type of lymphoma occurring in the breast. All tissue sections were reviewed independently and re-diagnosed by three hematopathologists (W.Z., W.L., and S.Z.) according to the World Health Organization classification of tumors of hematopoietic and lymphoid tissues (Revised 4th Edition, 2017) [1]. Detailed clinical data, such as age, sex, clinical course, symptoms, laboratory tests, imaging findings, and treatment details, were collected from electronic medical records. Follow-up data were obtained by telephone interviews and/or medical records. Overall survival (OS) was calculated from the date of diagnosis form a breast sample to the date of death or last followup. Primary ENKTL-Breast was diagnosed based on the diagnostic criteria of primary breast lymphoma proposed by Wiseman and Liao, and later modified by Hugh et al. as follows: (1) adequate pathological material was available for review; (2) radiologically, the breast was considered as the primary site or site of major manifestation of the lymphoma; and (3) there was no prior documentation of a similar histological type of lymphoma other than that of the ipsilateral axillary nodes [29-31]. The cases, which did not fulfill the criteria of primary ENKTL-Breast, were defined as secondary ENKTL-Breast.

\section{Histological assessment}

All tissue specimens were fixed with $10 \%$ formalin and embedded in paraffin after routine processing. Tissue Sects. $(3-4 \mu \mathrm{m})$ were stained with hematoxylin and eosin for subsequent microscopic examination.

\section{Immunohistochemistry analysis}

For the immunohistochemical analysis, the following lymphoma antibodies were used: cytoplasmic CD3 (cCD3, PS1, Dako, Glostrup, Denmark), CD20 (L26; Dako, Glostrup, Denmark), CD5 (4C7; Novocastra, Newcastle, UK), CD4 (RMA-0620; Maixin, Shenzhen, China), CD8 (C8; Maixin, Shenzhen, China), TIA-1 (2G9; Dako, Glostrup, Denmark), granzyme B (GZB01; Neomarkers, Fremont, CA, USA), and CD56 (123C3; Zymed, Guangzhou, China), CD30 (Ber-H2; Neomarkers, Fremont, CA, USA), and Ki-67 (M7259; Dako, Glostrup, Denmark). All immunostaining were performed as previously described [32], and appropriate positive and negative controls were employed. The positive rate of CD30 and Ki-67 (positive tumor cells/total tumor cells) was recorded.

\section{In situ hybridization}

EBV status was evaluated using in situ hybridization with a digoxin-labeled oligonucleotide probe complementary to two EBER, namely, EBER-1 and EBER-2 
(EBER1/2; Dako, NO. Y520001). This was performed as previously described [33].

\section{T-cell receptor gene rearrangement analysis}

$T R$ gene rearrangement was detected based on BIOMED-2 operating instructions. The analysis was performed as previously described [32].

\section{Abbreviations}

ENKTL: Extranodal natural killer/T-cell lymphoma; EBV: Epstein-Barr virus; OS: Overall survival; EBER: EBV-encoded small RNAs; TR: T-cell receptor; HBV: Hepatitis B virus.

\section{Supplementary Information}

The online version contains supplementary material available at https://doi. org/10.1186/s13023-021-02110-x.

Additional file 1: Table S1. Clinical features of ENKTL-breast queried in the literature. Table S2. Composition of breast lymphoma cases between 2010 and 2020 in our institution.

\section{Acknowledgements}

None.

\section{Authors' contributions}

ZHC and SZ conceived and designed the study. FLL contributed to the radiological imaging. WYZ, SZ and WPL made pathological diagnosis of the patients. WL analyzed the data and performed the literature review, then ZHC rechecked it. WL wrote the initial draft of the manuscript. SZ and ZHC revised it. All authors read and approved the final manuscript.

\section{Funding}

This study was supported by the National Natural Science Foundation of China (30900534, 81900195), 1·3.5 project for disciplines of excellence-Clinical Research Incubation Project, West China Hospital, Sichuan University (2021HXFH027), and Post-Doctor Research Project, West China Hospital, Sichuan University (2019HXBH067)

\section{Availability of data and materials}

All data generated or analyzed during the current study are included in this published article.

\section{Declarations}

Ethics approval and consent to participate

Not applicable.

\section{Consent for publication}

Not applicable.

\section{Competing interests}

The authors declare that they have no competing interests.

\section{Author details}

'Department of Pathology, West China Hospital, Sichuan University, No. 37 , GuoXue Xiang, Chengdu 610041, Sichuan, China. ${ }^{2}$ Department of Pathology, Fujian Medical University Cancer Hospital, Fujian Cancer Hospital, Fuzhou, China. ${ }^{3}$ Department of Nuclear Medicine, West China Hospital, Sichuan University, Chengdu 610041, China.

Received: 10 August 2021 Accepted: 6 November 2021 Published online: 18 November 2021
References

1. Chan JKC, Quintanilla-Martinez L, Ferry JA. Extranodal NK/T-cell lymphoma, nasal type. In: Steven H, Swerdlow EC, Harris NL, Jaffe ES, Pileri SA, Stein H, Thiele J, Arber DA, Hasserjian RP, Le Beau MM, Orazi A, Siebert $\mathrm{R}$, eds. WHO classification of tumours of haematopoietic and lymphoid tissues, Revised 4th ed. Lyon: IARC; 2017:p368-71.

2. Chan JK, Yip TT, Tsang WY, Ng CS, Lau WH, Poon YF, et al. Detection of Epstein-Barr viral RNA in malignant lymphomas of the upper aerodigestive tract. Am J Surg Pathol. 1994:18:938-46.

3. Arber DA, Weiss LM, Albújar PF, Chen YY, Jaffe ES. Nasal lymphomas in Peru. High incidence of T-cell immunophenotype and Epstein-Barr virus infection. Am J Surg Pathol. 1993;17:392-9.

4. Ho FC, Todd D, Loke SL, Ng RP, Khoo RK. Clinico-pathological features of malignant lymphomas in 294 Hong Kong Chinese patients, retrospective study covering an eight-year period. Int J Cancer. 1984;34:143-8.

5. Zhou ZJ, Xie JL, Wei P, Zhou XG. Pathologic subtyping of primary lymphoma of breast and prognostic analysis. Zhonghua Bing Li Xue Za Zhi. 2017:46:618-22.

6. Cho EY, Gong G, Khang SK, Kang YK, Huh J. Fine needle aspiration cytology of CD56-positive natural killer/T-cell lymphoma of soft tissue. Cancer. 2002;96:344-50.

7. Tsao L, Draoua HY, Mansukhani M, Bhagat G, Alobeid B. EBV-associated, extranodal NK-cell lymphoma, nasal type of the breast, after heart transplantation. Mod Pathol. 2004;17:125-30.

8. Aladily TN, Nathwani BN, Miranda RN, Kansal R, Yin CC, Protzel R, et al. Extranodal NK/T-cell lymphoma, nasal type, arising in association with saline breast implant: expanding the spectrum of breast implant-associated lymphomas. Am J Surg Pathol. 2012;36:1729-34.

9. Fréling E, Granel-Brocard F, Serrier C, Ortonne N, Barbaud A, Schmutz JL. Extranodal NK/Tcell lymphoma, nasal-type, revealed by cutaneous breast involvement (Article in French). Ann Dermatol Venereol. 2015;142:104-11.

10. Aguilera NS, Tavassoli FA, Chu WS, Abbondanzo SL. T-cell lymphoma presenting in the breast: a histologic, immunophenotypic and molecular genetic study of four cases. Mod Pathol. 2000;13:599-605.

11. Rahal A, Reddy PS, Alvares C. Extranodal NK/T-cell lymphoma, nasal type presenting as a breast mass. Cureus. 2015;7:e408

12. Li S, Feng X, LiT, Zhang S, Zuo Z, Lin P, et al. Extranodal NK/T-cell lymphoma, nasal type: a report of 73 cases at MD Anderson Cancer Center. Am J Surg Pathol. 2013;37:14-23.

13. Xiang CX, Chen ZH, Zhao S, Gao LM, Tao Q, Zuo Z, et al. Laryngeal extranodal nasal-type natural killer/T-cell lymphoma: a clinicopathologic study of 31 cases in China. Am J Surg Pathol. 2019:43:995-1004.

14. Huang Y, Shi X, Zhong P, Wang Y, Xiao H, Zhou X, et al. De novo testicular extranodal NK/T-cell lymphoma: a clinicopathologic study of 21 cases with review of additional 18 cases in the literature. Am J Surg Pathol. 2019:43:549-58.

15. Arber DA, Simpson JF, Weiss LM, Rappaport H. Non-Hodgkin's lymphoma involving the breast. Am J Surg Pathol. 1994;18:288-95.

16. Thomas A, Link BK, Altekruse S, Romitti PA, Schroeder MC. Primary Breast Lymphoma in the United States: 1975-2013. J Natl Cancer Inst. 2017;109:djw294.

17. Moura C, Leite MI, Parreira R, Medeiros A. Primary breast lymphoma. J Surg Case Rep. 2020;2020:riz405.

18. Talwalkar SS, Miranda RN, Valbuena JR, Routbort MJ, Martin AW, Medeiros LJ. Lymphomas involving the breast: a study of 106 cases comparing localized and disseminated neoplasms. Am J Surg Pathol. 2008:32:1299-309.

19. Avenia N, Sanguinetti A, Cirocchi R, Bistoni G, Trastulli S, D'Ajello F, et al. Primary breast lymphomas: a multicentric experience. World I Surg Oncol. 2010;8:53.

20. Domchek SM, Hecht JL, Fleming MD, Pinkus GS, Canellos GP. Lymphomas of the breast: primary and secondary involvement. Cancer. 2002;94:6-13.

21. Bagg A, Dunphy CH. Immunosuppressive and immunomodulatory therapy associated lymphoproliferative disorders. Semin Diagn Pathol. 2013;30:102-12.

22. Jonsson MV, Theander E, Jonsson R. Predictors for the development of non-Hodgkin lymphoma in primary Sjöogren's syndrome. Presse Med. 2012:41:e511-516.

23. Au WY, Weisenburger DD, Intragumtornchai T, Nakamura S, Kim WS, Sng I, et al. Clinical differences between nasal and extranasal natural killer/T-cell 
lymphoma: a study of 136 cases from the International Peripheral T-Cell Lymphoma Project. Blood. 2009;113:3931-7.

24. Yu BH, Shui RH, Sheng WQ, Wang CF, Lu HF, Zhou XY, et al. Primary intestinal extranodal natural killer/T-cell lymphoma, nasal type: a comprehensive clinicopathological analysis of 55 cases. PLoS ONE. 2016;11:e0161831.

25. Yu JB, Zuo Z, Tang Y, Zhao S, Zhang YC, Bi CF, et al. Extranodal nasal-type natural killer/T-cell lymphoma of the skin: a clinicopathologic study of 16 cases in China. Hum Pathol. 2009;40:807-16.

26. Kim SJ, Lim JQ, Laurensia Y, Cho J, Yoon SE, Lee JY, et al. Avelumab for the treatment of relapsed or refractory extranodal NKTT-cell lymphoma: an open-label phase 2 study. Blood. 2020;136:2754-63.

27. Kwong YL, Chan TSY, Tan D, Kim SJ, Poon LM, Mow B, et al. PD1 blockade with pembrolizumab is highly effective in relapsed or refractory NK/T-cell lymphoma failing l-asparaginase. Blood. 2017;129:2437-42.

28. Kawamoto K, Miyoshi H, Suzuki T, Sasaki Y, Yamada K, Yanagida E, et al. Frequent expression of CD30 in extranodal NK/T-cell lymphoma: potential therapeutic target for anti-CD30 antibody-based therapy. Hematol Oncol. 2018;36:166-73.

29. Wiseman C, Liao KT. Primary lymphoma of the breast. Cancer. 1972:29:1705-12
30. Hugh JC, Jackson Fl, Hanson J, Poppema S. Primary breast lymphoma. An immunohistologic study of 20 new cases. Cancer. 1990;66:2602-11.

31. Cheah CY, Campbell BA, Seymour JF. Primary breast lymphoma. Cancer Treat Rev. 2014;40:900-8.

32. Chen Z, Liu W, Zhang W, Ye Y, Guan P, Gao L, et al. Chronic active Epstein-Barr virus infection of T/NK-cell type mimicking classic Hodgkin lymphoma: clinicopathologic and genetic features of 8 cases supporting a variant With "Hodgkin/Reed-Sternberg-like" cells of NK phenotype. Am J Surg Pathol. 2019;43:1611-21.

33. Chen Z, Zou Y, Liu W, Guan P, Tao Q, Xiang C, et al. Morphologic patterns and the correlation with MYD88 L265P, CD79B mutations in primary adrenal diffuse large B-cell lymphoma. Am J Surg Pathol. 2020;44:444-55.

\section{Publisher's Note}

Springer Nature remains neutral with regard to jurisdictional claims in published maps and institutional affiliations.
Ready to submit your research? Choose BMC and benefit from:

- fast, convenient online submission

- thorough peer review by experienced researchers in your field

- rapid publication on acceptance

- support for research data, including large and complex data types

- gold Open Access which fosters wider collaboration and increased citations

- maximum visibility for your research: over $100 \mathrm{M}$ website views per year

At BMC, research is always in progress.

Learn more biomedcentral.com/submissions 